\title{
Implementation of Spatial Transformation Rules for Goal-Directed Reaching via Gain Modulation in Monkey Parietal and Premotor Cortex
}

\author{
Alexander Gail, Christian Klaes, and Stephanie Westendorff \\ Bernstein Center for Computational Neuroscience, German Primate Center-Leibniz Institute for Primate Research, 37077 Göttingen, Germany
}

\begin{abstract}
Planning goal-directed movements requires the combination of visuospatial with abstract contextual information. Our sensory environment constrains possible movements to a certain extent. However, contextual information guides proper choice of action in a given situation and allows flexible mapping of sensory instruction cues onto different motor actions. We used anti-reach tasks to test the hypothesis that spatial motor-goal representations in cortical sensorimotor areas are gain modulated by the behavioral context to achieve flexible remapping of spatial cue information onto arbitrary motor goals. We found that gain modulation of neuronal reach goal representations is commonly induced by the behavioral context in individual neurons of both, the parietal reach region (PRR) and the dorsal premotor cortex (PMd). In addition, PRR showed stronger directional selectivity during the planning of a reach toward a directly cued goal (pro-reach) compared with an inferred target (anti-reach). PMd, however, showed stronger overall activity during reaches toward inferred targets compared with directly cued targets. Based on our experimental evidence, we suggest that gain modulation is the computational mechanism underlying the integration of spatial and contextual information for flexible, rule-driven stimulus-response mapping, and thereby forms an important basis of goal-directed behavior. Complementary contextual effects in PRR versus PMd are consistent with the idea that posterior parietal cortex preferentially represents sensory-driven, "automatic" motor goals, whereas frontal sensorimotor areas are stronger engaged in the representation of rule-based, "inferred" motor goals.
\end{abstract}

\section{Introduction}

The same visual scene can lead to very different actions taken depending on the behavioral context. In a real boxing match, the face of your opponent is the direct goal of your arm movement. In a show fight, the face is not the goal of the movement but still guides it. Planning a reach toward a visual target object or inferring a reach goal from the position of an object according to a spatial transformation rule requires context-specific sensorimotor transformations (Gail and Andersen, 2006). Here, we test how the frontoparietal reach network flexibly creates context-specific motor-goal representations.

Spatially flexible cue-response mapping, as in a pro-reach/ anti-reach task, requires integration of spatial sensory information with the context. Computational models suggest that this flexibility can be achieved with contextual gain modulation of spatially selective neurons (Salinas, 2004; Brozović et al., 2007), equivalent to gain field mechanisms suggested for multisensory integration (Andersen et al., 1985; Zipser and Andersen, 1988; Boussaoud et al., 1993, 1998; Brotchie et al., 1995; Galletti et al.,

Received March 5, 2009; revised June 13, 2009; accepted June 16, 2009.

This work was supported by the Federal Ministry for Education and Research (Germany) Grants 01600433 and 01GQ0814. We thank Sina Plümer and Ludwig Ehrenreich for their extensive support in data collection, and Axel Lindner for helpful discussions on previous versions of this manuscript.

Correspondence should be addressed to Dr. Alexander Gail, Bernstein Center for Computational Neuroscience, German Primate Center-Leibniz Institute for Primate Research, Kellnerweg 4, 37077 Göttingen, Germany. E-mail: agail@gwdg.de.

D0I:10.1523/JNEUROSCI.1095-09.2009

Copyright $\odot 2009$ Society for Neuroscience $\quad$ 0270-6474/09/299490-10\$15.00/0
1995; Salinas and Abbott, 1996; Snyder et al., 1998; Salinas and Thier, 2000). If the idea of gain modulation for space-context integration holds true, then spatially selective neurons in primate cortical sensorimotor areas, like the parietal reach region (PRR) and the dorsal premotor cortex (PMd), should be upregulated and downregulated by the behavioral context.

Spatial sensory information presumably reaches the frontoparietal sensorimotor network via the posterior parietal cortex (Colby et al., 1988; Blatt et al., 1990; Marconi et al., 2001). Associative goal selection criteria or arbitrary transformation rules are believed to exert their influence on motor planning via prefrontal and premotor areas (Rushworth et al., 1997; Wise and Murray, 2000; Toni et al., 2001; Wallis and Miller, 2003). Both PMd and PRR have been shown to express sustained spatially selective activity during movement planning (Weinrich and Wise, 1982; Andersen et al., 1985; Boussaoud and Wise, 1993; Kalaska, 1996; Wise et al., 1997; Andersen and Buneo, 2002; Cisek, 2007), predominantly representing motor goals (di Pellegrino and Wise, 1993; Crammond and Kalaska, 1994; Gail and Andersen, 2006). We compare contextual modulations in PRR and PMd to test whether the two areas represent inferred versus directly cued motor goals differently. Posterior parietal cortex, for example, was hypothesized to mainly represent fast "automatic" motor goals (Desmurget et al., 1999; Pisella et al., $2000)$, as in pro-reaches.

We tested whether and how motor-goal representations in PRR and PMd are modulated by context-specific spatial transformation rules during reach planning. We used a memory-guided 
a

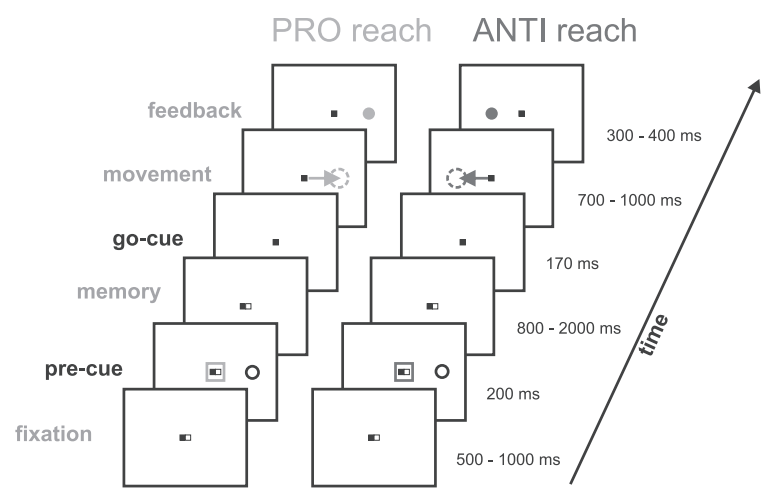

b

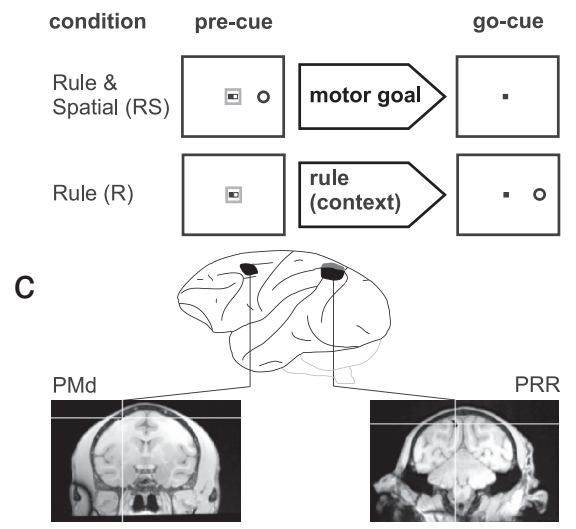

Figure 1. Memory-guided anti-reach task with precuing. $\boldsymbol{a}$, The left sequence shows an example of a pro-trial, and the right, an example of an anti-trial. Subjects had to maintain eye fixation (central dark spot) throughout the trial and hand fixation (central white spot) until the go instruction (disappearance of the white spot). The reach goal was defined by the combination of a context cue (colored central frame), indicating the pro/anti transformation rule, and a color-neutral spatial cue, which was presented at any of four different peripheral screen positions $\left(0,90,180,270^{\circ}\right)$. Cues could be presented before (precue period) or after a variable memory period (go cue period), simultaneously or separately. In the example, the context and the spatial cue are both presented during the precue period. To be rewarded, the subject had to make a reach toward the previous spatial cue position (pro-reach) or to the opposite side (anti-reach). The arrows and dotted circles are for demonstration purpose only and were not shown to the subjects. $\boldsymbol{b}$, In this study, we compare conditions in which the subjects either had full information on the upcoming motor goal (transformation rule and spatial cue presented as precue; RS condition) or only the context information available (transformation rule presented as precue, spatial cue during go cue period; R condition). c, Extracellular signals from multiple individual neurons were recorded simultaneously in PRR and PMd (regions of interest for monkey $S$ ) while the monkeys performed the task.

pro-reach/anti-reach task with partial precuing to investigate contextual modulations that were either independent or dependent of the spatial tuning. We tested separately the effect of context on either the response amplitude or the directional selectivity. Our results are consistent with the idea of space-context integration by a gain modulation mechanism. Additionally, we found complementary effects of context on motor-goal representations in PRR and PMd, which indicate different mutual roles of the two areas in context-specific visuomotor integration and motor-goal representation.

\section{Materials and Methods}

Tasks and control of behavioral parameters. Two male rhesus monkeys (Macaca mulatta; S and A) were trained to perform a memory-guided anti-reach task with partial precuing (Fig. 1a). Two visual cues, one spatial and one contextual, were presented to the subjects either simultaneously or at different points in time (19 inch ViewSonic LCD VX922; $5 \mathrm{~ms}$ off-on-off response time). The contextual cue (transformation rule) consisted of a green (pro-reach) or blue (anti-reach) frame around the eye and hand fixation points and instructed the subject to reach either toward (pro) or diametrically opposite to the spatial cue (anti). The peripheral spatial cue was located at one of four possible positions $(0,90$, 180 , and $270^{\circ}$ ) with an eccentricity of $9 \mathrm{~cm}\left[14.5^{\circ}\right.$ visual angle (VA)] relative to the fixation point. The motor goal was only defined uniquely, once both cues were known to the subject.

The monkey initiated a trial, by fixating a small red square in the center of the screen (eye fixation tolerance, $2.5-4.0^{\circ} \mathrm{VA} ; 224 \mathrm{~Hz} \mathrm{CCD}$ camera; ET-49B; Thomas Recording) and touching an adjacent white square of the same size (hand fixation tolerance: $4.0^{\circ} \mathrm{VA}$, touch screen mounted directly in front of the video screen; IntelliTouch; ELO Systems). After a short period of 500-1000 ms (fixation period), the precue appeared for $200 \mathrm{~ms}$. The precue could be either the contextual and the spatial cue at once [rule and spatial condition (RS)], only the spatial cue [spatial-only condition (S)], only the contextual cue [rule-only condition (R)], or nothing [null condition $(\mathrm{N})$ ], leading to four different memory conditions. After the precue, the monkey had to keep eye and hand fixation for $800-2000 \mathrm{~ms}$ (memory period). Depending on the memory condition, additional information was given to the monkey at the end of memory period. In the $\mathrm{R}, \mathrm{S}$, and $\mathrm{N}$ conditions, the missing information required to fully specify the reach goal (the spatial cue, the contextual cue, or both) was briefly flashed during the $170 \mathrm{~ms}$ go cue period. In the RS condition, no additional information was shown. Simultaneously, with the presentation of the second cue, the hand fixation square disappeared ("go" signal) and the monkey had to reach toward the instructed goal within a maximum of $700-1000 \mathrm{~ms}$ (movement period; $4.9^{\circ} \mathrm{VA}$ reach tolerance) and hold the goal position for $300-400 \mathrm{~ms}$ (feedback period). The monkey received visual feedback about the correct movement goal, which consisted of a filled circle of the same color as the contextual cue and was presented at the goal location during the feedback period. Eye fixation had to be kept throughout the trial; otherwise, the trial was aborted immediately. Liquid reward and acoustic feedback indicated correct (high pitch tone, reward) or incorrect (low pitch tone, no reward) behavior.

Pro-trials and anti-trials were randomly interleaved from trial to trial ( $\sim 400$ trials per data set) and the four memory conditions were randomly mixed in small blocks (10-20 trials of same condition per block). To test the contextual influence on motor planning, only the results of the RS and R conditions (transformation rule available during the planning phase of the movement) are meaningful and presented here (Fig. 1b).

Animal preparation and neural recordings. After training of the task, both monkeys were implanted with titanium head holders that were custom-fit to their heads based on computer-tomographical surface reconstruction of the skull (3di $\mathrm{GmbH})$. After recovery and additional training, each monkey was implanted with two magnetic resonance imaging (MRI)-compatible recording chambers, also custom-fit to the skull. One chamber was placed above PRR [Horsley Clarke coordinates: $6 \mathrm{~mm} /-8.5 \mathrm{~mm}$ (monkey S/A) lateral; $-10 \mathrm{~mm} /-9.5 \mathrm{~mm}$ anterior], the other chamber above PMd (13 mm/-13.5 mm lateral; $17 \mathrm{~mm} / 19.8 \mathrm{~mm}$ anterior). Presurgical structural MRI was used to position the chambers (Fig. 1c). Postsurgical MRIs, showing the chamber relative to the brain, verified the correct chamber positions and allowed precise targeting of the desired anatomical structure. Sustained, direction-selective, neural responses during center-out reach planning was used as a physiological signature in both areas to confirm the imaging-based positioning. Both chambers were implanted contralaterally to the handedness of the monkey (A, left hemisphere; $\mathrm{S}$, right hemisphere). All surgical and imaging procedures were conducted under general anesthesia.

For the extracellular recordings, up to four microelectrodes in each cortical area in a five-channel microdrive ("mini-matrix"; Thomas Recording) were used simultaneously. In most sessions, simultaneous recordings were conducted in both areas. The raw signals were preamplified (20×; Thomas Recording), bandpass filtered, and amplified (154 
$\mathrm{Hz}$ to $8.8 \mathrm{kHz}$; $400-800 \times$; Plexon), before on-line spike-sorting was conducted (Sort Client; Plexon). In addition to spike times, the spike waveforms were recorded and later subjected to off-line sorting for optimal isolation quality (Offline Sorter; Plexon).

Animal care and all experimental procedures were conducted in accordance with German law (Animal Welfare Act).

Selection of neural data. All recorded and sufficiently well isolated units, regardless of their tuning properties, were included in the neural data analyses unless explicitly stated otherwise. We analyzed the neural activity during the last $300 \mathrm{~ms}$ of the memory period (i.e., activity succeeding the precue with a time lag of at least $500 \mathrm{~ms}$, and immediately preceding the go cue). This period was chosen to extract movement planning activity without confounding effects of (1) immediate visual input from the cue stimuli, or (2) transition phases from visual to motor-goal tuning (Gail and Andersen, 2006), or (3) visual and somatosensory input and motor-control signals related to movement initiation.

Analyses of neuronal directional selectivity. Directional selectivity was quantified with a directional tuning vector (DTV), which is defined as the vector average across all center-out cue directions $\vec{u}_{i}$ (unit vectors) weighted with the corresponding mean neural spike rates across trials with this cue direction $\left(r_{i j}\right)$ and normalized to the total mean spike rate across all trials for the neuron $j$ as follows:

$$
\operatorname{DTV}_{j}=\sum_{i=1}^{4} r_{i j} \vec{u}_{i} / \sum_{i=1}^{4} r_{i j}
$$

The length of the resulting vector is between 0 and 1 and is a measure of the tuning strength. Its direction will be referred to as preferred direction (PD) of a neuron. The DTV was computed separately for pro-trials and anti-trials and was defined relative to the position of the spatial cue, not relative to the movement goal.

Significance of the directional selectivity was tested with a nonparametric one-way ANOVA (Kruskal-Wallis; Matlab; Mathworks) with the four different visual cue directions as groups and sample sizes defined by the number of identical trial repetitions. The ANOVA was calculated independently within each transformation rule (pro/anti) and precuing condition (total of $2 \times 4=8$ combinations).

The relative difference in PD between pro-trials and anti-trials in the RS condition indicates whether the tuning of a neuron in the given time window reflects the visual cue or the reach goal position. We quantified this difference with the tuning direction difference index (DD) as follows:

$$
\mathrm{DD}=\mathrm{PD}_{A}-\mathrm{PD}_{P}
$$

$\mathrm{PD}_{P}$ and $\mathrm{PD}_{A}$ are the preferred directions of a neuron in pro-trials and anti-trials, respectively. A DD of $\pm 0^{\circ}$ indicates idealized visual tuning. A $\mathrm{DD}$ of $\pm 180^{\circ}$ means opposing PDs (relative to the cue position), which indicates idealized motor-goal tuning.

Analyses of contextual modulations. We tested three different effects of the context on the neuronal activity and quantified each of them with a contextual modulation index: gain modulation of spatially selective neurons (i.e., amplitude changes independent of changes in directional selectivity [gain modulation (GM)], changes in directional selectivity independent of changes in overall neural response strength [selectivity modulation (SM)], and direct modulation of neuronal activity levels independent of spatial tuning [direct modulation (DM)]).

Contextual gain modulation is characterized by amplitude changes of spatial tuning, independent of changes in directional selectivity. It is defined as follows:

$$
\mathrm{GM}=\frac{r_{P}^{\mathrm{RS}}-r_{A}^{\mathrm{RS}}}{r_{P}^{\mathrm{RS}}+r_{A}^{\mathrm{RS}}}
$$

where $r_{P}^{\mathrm{RS}}$ and $r_{A}^{\mathrm{RS}}$ are the mean spike rates for pro-trials and anti-trials in the RS condition. Since most neurons in PRR and PMd are spatially highly selective with a DD of around $\pm 180^{\circ}$ (see Results), large absolute GM values indicate strong gain modulation effects of the spatial motorgoal tuning by the context.
Contextual selectivity modulation is characterized by changes in directional selectivity independent of changes in response amplitude. To quantify contextual selectivity modulation, we computed the contrast of the normalized DTV length between pro-trials and anti-trials in the RS condition as follows:

$$
\mathrm{SM}=\frac{\left|\mathrm{DTV}_{P}\right|-\left|\mathrm{DTV}_{A}\right|}{\left|\mathrm{DTV}_{P}\right|+\left|\mathrm{DTV}_{A}\right|},
$$

where $\mathrm{DTV}_{P}$ and $\mathrm{DTV}_{A}$ are the normalized directional tuning vectors for pro-trials and anti-trials. A positive SM indicates that a neuron is more strongly tuned in pro-trials than in anti-trials, and vice versa for negative values. Values close to zero indicate that tuning selectivity is not modulated by the transformation rule.

Both GM and SM quantify modulations of spatial tuning by context. GM and SM are independent of each other in that each can change without affecting the other by varying parameters of hypothetical tuning functions (supplemental material S1, available at www.jneurosci.org). This does not mean that a single cell could not simultaneously have a high GM and SM. In fact, for neurons that are very strongly modulated by the context the activity in one of the two conditions (pro or anti) may be so weak that the tuning becomes arbitrarily shaped, which can lead to large GM and SM values and "random" DD values.

Direct context modulation can be quantified by differences in the memory activity between pro-reaches and anti-reaches in the R condition, since these differences reflect contextual influence independent of any spatial tuning. We defined the direct context modulation as the contrast of the average activities between pro-trials and anti-trials as follows:

$$
\mathrm{DM}=\frac{r_{P}^{R}-r_{A}^{R}}{r_{P}^{R}+r_{A}^{R}} .
$$

$r_{P}^{R}$ and $r_{A}^{R}$ are the mean spike rates in the $\mathrm{R}$ condition for pro-trials and anti-trials, respectively. A positive DM indicates neurons that are more active in pro-trials. A negative DM indicates stronger activity in anti-trials. Values close to zero indicate neurons indifferent to the transformation rule.

Note that all modulation indices are nonparametric and do not require fitting of any predefined tuning functions.

All contextual modulations were statistically analyzed on the level of individual neurons and, additionally, on the population level. We used bootstrap methods ( $n=100$ samples) to estimate the confidence limits of all indices (GM, SM, DM, and DD) for each individual neuron. The trial-by-trial spike rates were randomly permuted with repetitions, whereas the assignment of each trial to a certain direction, transformation rule, and memory condition was kept unchanged. GM, SM, and DM were considered significant if the $95 \%$ confidence limit did not overlap with zero. DD was considered to significantly deviate from motor-goal tuning if the circular confidence interval did not overlap with $180^{\circ}$.

On the population level, we estimated the to-be-expected distributions of all indices when assuming random data (shuffle test; $n=100 *$ number of used neurons). We randomly permuted trial-by-trial spike rates across the two transformation rules, while keeping the cue direction and memory condition unchanged. This procedure eliminated any effect of the transformation rule. For the GM, SM, and DM, $t$ tests were used to quantify deviations of the population means from zero. Additionally, a Bartlett test was used to test for deviations of the original distribution's variance from the normally distributed shuffled data. A positive Bartlett test indicates contextual modulations beyond what is expected because of random variations, even if the distribution of indices is centered at zero (i.e., even when there is no bias of the index toward either propreference or antipreference). To test whether a distribution of DD across the population of neurons deviates significantly from the predefined $\pm 180^{\circ}$ direction, we used circular statistics to compute the $95 \%$ confidence limits according to the following:

$$
d=\arccos \left[\frac{\sqrt{\frac{2 n\left(2 R^{2}-n \chi_{\alpha, 1}^{2}\right)}{4 n-\chi_{\alpha, 1}^{2}}}}{R}\right],
$$

where $R=n r . n$ is the number of samples, $r$ is the mean resultant vector length of the angular direction data (here, DD indices), and $\chi$ is the 
a

Classical motor goal cell $\mathrm{SM}=0.07, \mathrm{GM}=-0.02(P M d$, monkey $S)$

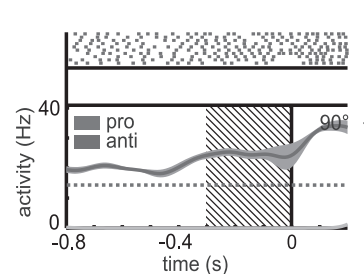

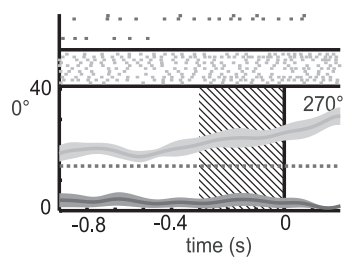

b

Relative tuning direction difference (DD)

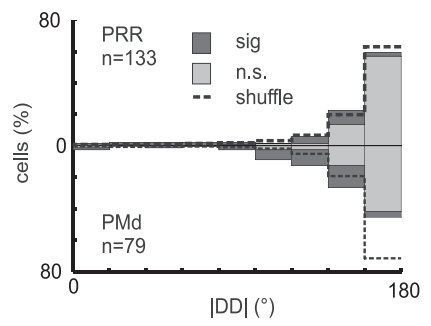

Figure 2. Motor-goal tuning in PRR and PMd during the late planning phase of reach movements. The example cell (a) shows classical motor-goal tuning, characterized by a PD depending on the motor goal and not the cue (DD not significantly different from $180^{\circ}$ ), as shown in the polar plot (middle). The response strength and selectivity were independent of the behavioral context (GM and SM not different from zero). Raster plots and spike density functions for pro (light gray) and anti (dark) trials are shown for the two most active directions (here, 90 and $270^{\circ}$ ) during the late memory period. Time 0 marks the onset of the go cue period. The mean firing rate during the fixation period is provided for comparison (dashed line). Spike density curves are smoothed with a Gaussian kernel ( $\sigma=50 \mathrm{~ms}$; dark lines, mean; light area, SE) for presentation purposes only. Also, polar tuning functions are interpolated for presentation purposes only, whereas all analyses are conducted nonparametrically on the original spike data. $\boldsymbol{b}$, Tuning DDs in PRR (top) and PMd (bottom) indicate that neurons in these areas are preferentially motor-goal tuned. Most individual neurons do not deviate significantly from $180^{\circ}$ (light gray bars); a few do according to their bootstrap confidence limits (dark bars). The circular distributions of the DD for all neurons in each area do not significantly deviate from $180^{\circ}$. Note that because of symmetry the distribution of absolute values (|DD|) are plotted, whereas circular statistics are applied to the original DD values.

inverse of the $\chi^{2}$ cumulative distribution function with a confidence limit of $1-\alpha$ (Zar, 1999).

To quantify the correlation between the signs and strengths of different forms of contextual modulation in individual neurons, we computed Pearson's cross-correlation coefficients of the different indices across the population of neurons. To make the modulation indices scale-invariant, we normalized the modulation indices to the variances of their respective distributions as follows:

$$
z_{i}=\frac{x_{i}}{\sigma_{i}}
$$

Here, $i$ is the considered index (GM, SM, DM), $x$ is the value of the index, and $\sigma$ is the variance of the index distribution over the cell population. Note that this normalization is only for easier comparison of the modulation strengths in the scatter plots of Figure 7. The cross-correlation coefficient is independent of this linear scaling, which is applied within each index distribution. To test whether different types of modulation tend to occur in complementary neuronal subpopulations, regardless of the "direction" of modulation (i.e., propreference vs antipreference), we also compared the absolute values of the different modulation indices. We used Spearman's rank correlation to account for the non-normal distribution of absolute modulation indices.

\section{Results}

Both monkeys performed the task with high accuracy. The percentage of overall correct trials was 78\% (monkey S) and $86 \%$ (monkey A) in the RS condition, and 75\% (S) and $83 \%$ (A) in the $\mathrm{R}$ condition. Error trials were mainly attributable to early trial abortion (mostly breaks in ocular fixation) but not attributable to a confusion of the "pro" and "anti" rules. The percentage of correct pro-reach and anti-reach decisions in otherwise-correct trials was 98\% (S) and 99\% (A) in the RS condition, and 94\% (S) and $99 \%$ (A) in the R condition. The performance of both monkeys showed no significant difference between pro-trials and anti-trials in the RS condition ( $p>0.05$, paired $t$ test), and a minimal difference in the $\mathrm{R}$ condition ( $\mathrm{S}: m_{\text {pro }}=95 \%, m_{\text {anti }}=94 \%, p=$ 0.008; A: $m_{\text {pro }}=99 \%, m_{\text {anti }}=98 \%, p=0.003$, paired $t$ test).

A total of 258 neurons from PRR (monkey S, 99; A, 159) and 193 from PMd (S, 75; A, 118) were recorded. Regardless of their spatial tuning properties, we tested all recorded neurons for direct context modulation (i.e., modulations of neuronal response amplitude by the context independent of directional selectivity) during the late memory period in the R condition (DM) (see Materials and Methods). For analyzing the effect of context on directional tuning properties (GM and SM) (see Materials and Methods and supplemental material S1, available at www. jneurosci.org, for details on modulation indices), we used a subset of neurons that had significant directional selectivity in at least one of the two spatial transformation conditions (pro/anti) during the late memory period of the RS condition. A total of 205 (79\%) neurons in PRR [S, 71 (71\%); A, $134(84 \%)]$ and 132 (68\%) neurons in PMd [S, 56 (75\%); A, 76 (64\%)] fulfilled this criterion. Data from both monkeys showed the same results and will be presented jointly (for comparison of the two monkeys, see supplemental material S2, available at www.jneurosci.org).

\section{Contextual gain modulations of motor-goal tuning}

When the reach goal was known to the monkeys during the memory period ( $\mathrm{RS}$ condition), most of the spatially selective neurons in PRR (82.0\%) and PMd (72.2\%) were tuned for the motor goal, as was expected from previous results (Crammond and Kalaska, 1994; Gail and Andersen, 2006). Some individual neurons in the current study showed "classical" motor-goal tuning, which was independent of the spatial transformation rule that led to this motor goal (i.e., independent of the context). In the example neuron (Fig. 2a), the preferred directions for procondition and anticondition were opposite to each other when measured relative to the position of the cue, which means they were aligned with the motor goal (tuning direction difference DD not different from $180^{\circ} ; p>0.05$; bootstrap test) (see Materials and Methods), and their response strength as well as the directional selectivity did not differ between the pro and the anti condition (GM and SM not different from $0 ; p>0.05$; bootstrap test) (see Materials and Methods and below). The population of neurons in both areas on average represented the direction of the motor goal and not the cue position. In other words, the distribution of DD across the population of neurons did not deviate from $180^{\circ}$ neither in PRR (circular mean, $179 \pm 8^{\circ}$ SEM) (see Materials and Methods) nor in PMd (mean, $184 \pm 10^{\circ}$ ) (Fig. $2 b$ ). To be included in this analysis, the cells had to be significantly tuned in both spatial transformation conditions (pro/anti). This criterion was met by 133 (52\%) of all neurons in PRR [S, 50 (51\%); A, 83 $(52 \%)]$ and 79 (41\%) of all neurons in PMd [S, 35 (47\%); A, 44 (37\%)].

To test for contextual effects on spatial tuning properties, we distinguished between contextual GM and contextual SM (see 
a pro-preferring motor goal cell $\mathrm{SM}=0.09, \mathrm{GM}=0.36(P M d$, monkey $A)$
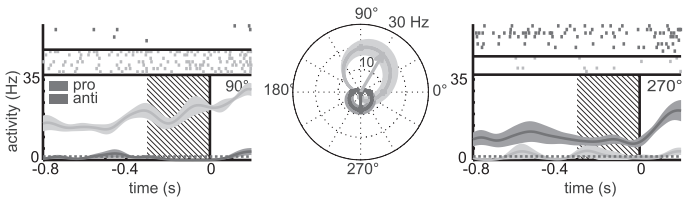

\section{anti-preferring motor goal cell} $\mathrm{SM}=0.05, \mathrm{GM}=-0.38(P R R$, monkey $A)$

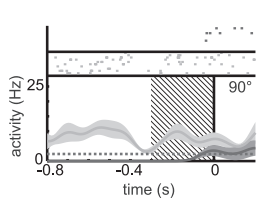

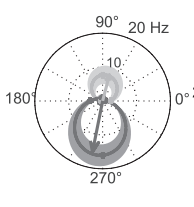

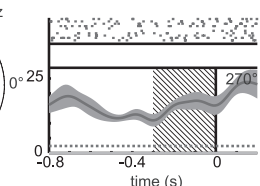

b

Gain modulation (GM)

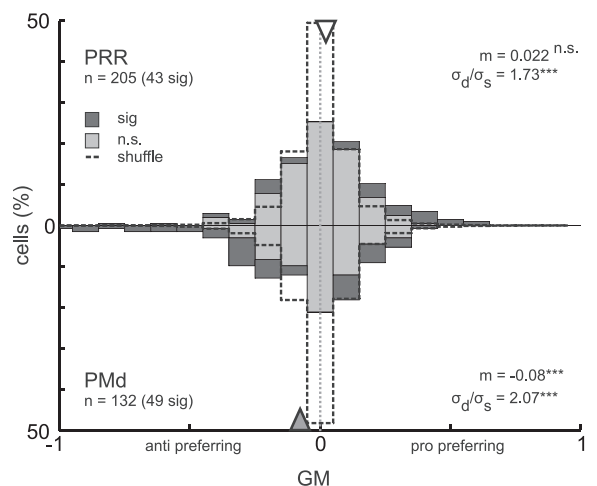

Figure 3. Contextual gain modulation of motor-goal tuning in PRR and PMd. $\boldsymbol{a}$, Examples of neurons with a high absolute GM indicating stronger activity for either pro-reaches (top) or anti-reaches (bottom), with invariant motor-goal tuning preference (DD not significantly different from $180^{\circ}$ ) and selectivity (SM not significantly different from zero; conventions are as in Fig. $2 a$ ). The anti-preferring neuron (bottom) was also one that fulfilled the strict criteria for an ideal contextual gain modulation of motor-goal tuning: the neuron was significantly tuned in pro and anti trials, had a significant GM, the DD was not significantly different from $180^{\circ}$, and the SM not significantly different from zero. (Note that the tuning vectors shown in the polar plot are not normalized, resulting in unequal length for pro and anti trials despite equally strong tuning; the SM is computed with normalized tuning vectors.) $\boldsymbol{b}$, Distribution of GM across the neuronal populations (conventions are as in Fig. $2 b$ ). In PMd (bottom), there was a significant bias toward negative values (mean, -0.08 ; gray triangle; $t$ test) indicating on average stronger antipreference in PMd, but not in PRR (top; white triangle). The GM distributions in both areas were broader than for the shuffled data (dashed line; $\sigma_{\mathrm{d}}>\sigma_{\mathrm{s}}$; Bartlett's test) indicating significant modulation effects in both areas. Significance levels were as follows: ${ }^{*} p<0.05,{ }^{* *} p<0.01,{ }^{* *} p<0.001$.

Materials and Methods and supplemental material S1, available at www.jneurosci.org). Figure $3 a$ shows two example neurons that were gain modulated by the behavioral context. The first neuron (top) was highly active in pro-trials, but responded only weakly in anti-trials. The preferred direction, representing the motor goal, remained unchanged (DD not significantly different from $180^{\circ}$ ), as did the directional selectivity (SM not different from zero). The second neuron (bottom row) showed the opposite preference and responded strongly in anti-trials, but only weakly in pro-trials. Again the spatial tuning encoded the motor goal in both rule conditions in a similar manner. Twenty-one percent (43 of 205) of neurons in PRR and 37\% (49 of 132) in PMd had a significant GM when tested in individual neurons (Fig. $3 b$ ). There was a significant difference between the numbers of significant pro-preferring and anti-preferring neurons in PRR (31 pro, 12 anti; $p=3.7 \times 10^{-3} ; \chi^{2}$ test) but not in $\operatorname{PMd}(28: 21$; $p>0.05$ ) (for the number ratios, see Fig. $6 c$ ). On the population level, in both areas the GM values were more broadly distributed (i.e., on average, had higher absolute values) than the GM of the shuffled data (PRR, $p<10^{-10}$; PMd, $p<10^{-10}$; Bartlett's test), indicating contextual gain modulation (Fig. 3b) (see also Fig. 6a). In PMd, the GM distribution additionally had a significant bias toward anti-preferring neurons $\left(m=-0.08 ; p=8 \times 10^{-4} ; t\right.$ test) (i.e., neurons in PMd were on average more active during the planning of anti-reaches). In PRR, the GM distribution was centered on zero $(m=0.021 ; p>0.05 ; t$ test) (see also Fig. $6 b)$.

\section{Contextual modulations of directional selectivity}

In addition to a modulatory effect on the gain of the motor-goal tuning, context also modulated the directional selectivity of many neurons in our experiment (Fig. 4). In the first example (Fig. 4a, top), the tuning of the neuron, which was highly selective for pro-reaches to the left, became bimodal in the case of antireaches (i.e., was active for leftward and rightward anti-reaches). The second example (Fig. 4a, bottom) shows another neuron that was spatially tuned in pro-trials but not tuned in anti-trials. Twenty-six percent (54 of 205) of neurons in PRR and 25\% (33 of 132 ) in PMd had a significant SM (Fig. 4b). There was a signifi- cant difference between the numbers of significant propreferring and anti-preferring neurons in PRR (45 pro, 9 anti; $p=3.2 \times 10^{-7} ; \chi^{2}$ test) but not in PMd $(18: 15 ; p>0.05)$ (see also Fig. $6 c$ ). Correspondingly, the population distribution of SM (Fig. $4 b)$ in PRR shows a shift toward positive values $(m=0.11 ; p=2.0 \times$ $10^{-7} ; t$ test), indicating a bias toward greater directional selectivity in pro-trials than in anti-trials (see also Fig. 6b). In PMd, there was no shift of the population mean toward pro-preferring or antipreferring selectivity ( $m=0.02 ; p>0.05 ; t$ test). SMs were more widely distributed in the original data than in the shuffled data in both areas (Bartlett's test: PRR, $p=2.4 \times 10^{-4}$; PMd, $p=4.7 \times$ $\left.10^{-6}\right)$, indicating significantly stronger modulation of tuning selectivity than expected by chance (see also Fig. $6 a$ ). The SM values in PMd had a trend to be bimodally distributed, indicating simultaneous presence of neurons with rather strong modulation of directional selectivity preferring either pro-reaches or anti-reaches.

\section{Direct context modulation}

Modulation of motor-goal tuning in $\mathrm{PMd}$ and $\mathrm{PRR}$ requires that the contextual information about the currently valid transformation rule is accessible to these cortical regions. How strongly is this transformation rule represented in PRR and PMd neurons at a time when only the rule (pro/anti) and not the complete motor goal is known to the monkey (i.e., independent of any spatial representations)? The direct context modulation index (DM) compares the level of neuronal activity during the memory period in the $\mathrm{R}$ condition, when contextual but not spatial information is available. Sixteen percent (42 of 258) of neurons in PRR and 25\% (49 of 193) in PMd had a significant DM (Fig. 5). There was no significant difference between the numbers of significant pro-preferring and anti-preferring neurons in either PRR (24 pro, 18 anti; $p>0.05 ; \chi^{2}$ test) or PMd (23:26; $\left.p>0.05\right)$ (Fig. $6 c$ ). The balance between propreference and antipreference was also reflected in the fact that the distributions of DM values were centered at zero in PRR (mean $m=0.011 ; p>0.05 ; t$ test) and PMd $(m=0.013 ; p>0.05)$ (Fig. $6 b)$. The variances of the DM distributions were significantly larger than the variances of the 
a Pro selective cell (bimodal in anti) $\mathrm{SM}=0.73, \mathrm{GM}=-0.06(P R R$, monkey $A)$
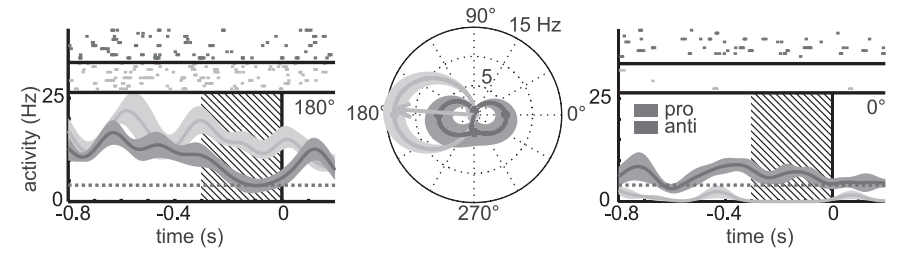

Pro selective cell (untuned in anti) $\mathrm{SM}=0.82, \mathrm{GM}=0.01(P R R$, monkey $A)$
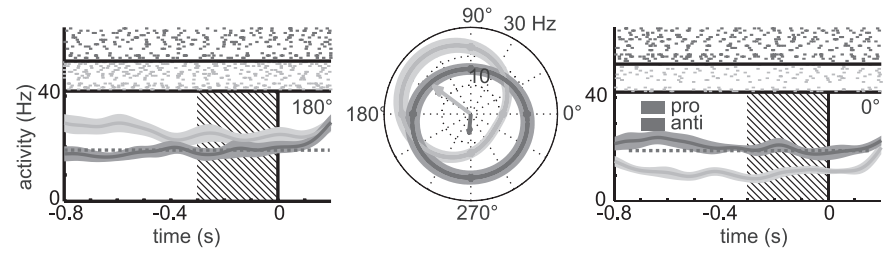

b Selectivity modulation (SM)

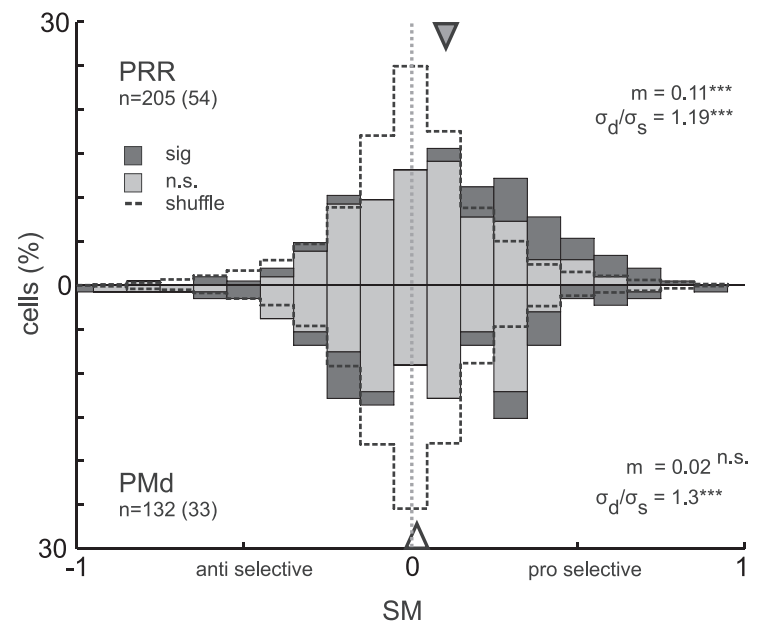

Figure 4. Contextual selectivity modulation of motor-goal tuning in PRR and PMd. $\boldsymbol{a}$, Examples of cells with a high absolute SM, indicating stronger directional selectivity for either pro-reaches or anti-reaches, but having the same response strength for both conditions on average across all reach directions (GM not significantly different from zero; conventions are as in Fig. 2a). The modulation resulted in different forms of tuning [e.g., bimodal (top) or nonselective (bottom) tuning functions during anti-reach planning]. $\boldsymbol{b}$, Distribution of SM across the neuronal populations (conventions are as in Fig. 2b). There was a significant bias for positive (pro-preferring) values in PRR (top), but not in PMd (bottom), indicating on average stronger directional selectivity during pro-reach planning in PRR, like the examples shown in $\boldsymbol{a}$. The SM distributions in both areas were broader than those of the shuffled data (dashed line; $\sigma_{\mathrm{d}}>\sigma_{\mathrm{s}^{\prime}}$ Bartlett's test) indicating significant modulation effects in both areas (conventions are as in Fig. 3).

shuffled DM data in both areas (PRR, $p=9.1 \times 10^{-4} ; \mathrm{PMd}, p=$ $1.1 \times 10^{-7}$; Bartlett's test) (Fig. $6 a$ ).

\section{Complementary contextual modulations in PRR and PMd}

Figure 6 summarizes the contextual modulation effects on the gain of tuned neural responses (GM) and their directional selectivity (SM), together with the direct neural responses to the context information itself (DM). When compared with the shuffle prediction, contextual gain modulation showed the strongest effect, both in PRR and PMd (Fig. 6a). Yet directional selectivity modulation and direct contextual modulation indices were also significantly larger than expected by chance in both areas. The bias of the contextual modulation effects in terms of a propreference/antipreference were complementary in the two areas (Fig. 6b): Contextual gain modulations in PMd were biased toward antipreference [i.e., tuned responses were on average $15 \%$ $(\mathrm{GM}=-0.08)$ higher during anti-reaches compared with proreaches, whereas in PRR gain modulations on average were balanced]. In contrast, directional selectivity modulations in PRR were biased toward propreference; i.e., spatial tuning was on average stronger in pro-reaches compared with anti-reaches by $20 \%(\mathrm{SM}=0.11)$, whereas in PMd, selectivity modulations on average were balanced. In PRR, the fraction of individual neurons being biased toward higher response amplitudes or stronger directional selectivity was larger in pro-reaches compared with anti-reaches, whereas in PMd the number of individually modulated neurons was balanced (Fig. $6 c$ ).

\section{Different types of modulation in different neuronal populations?}

The contextual gain and selectivity modulation indices (GM and SM) per se are independent of each other (supplemental material S1, available at www.jneurosci.org). But one could imagine that, in individual neurons, the different modulation effects are correlated. This would be the case if two types of modulation are the phenomenological consequences of one and the same underlying computational mechanism, or if the underlying processes causing the different types of modulation interfere with each other. There was no correlation in either PRR or in PMd between GM and DM $\left(r_{\mathrm{PRR}}=0.1, p_{\mathrm{PRR}}>0.05 ; r_{\mathrm{PMd}}=0.03, p_{\mathrm{PMd}}>0.05\right), \mathrm{SM}$ and DM $\left(r_{\mathrm{PRR}}=0.13, p_{\mathrm{PRR}}>0.05 ; r_{\mathrm{PMd}}=0.07, p_{\mathrm{PMd}}>0.05\right)$, or $\mathrm{SM}$ and $\mathrm{GM}\left(r_{\mathrm{PRR}}=0.06, p_{\mathrm{PRR}}>0.05 ; r_{\mathrm{PMd}}=0.03, p_{\mathrm{PMd}}>0.05\right)$ (Fig. $7 a-c$ ).

Two reasons could account for a lack of correlation between GM, SM, and DM. Either there was no interaction between the underlying processes that caused the gain and the selectivity modulations, but they still occurred within the same neurons; or these processes affected complementary neuronal subpopulations. If different neuronal subpopulations were affected by contextual gain and selectivity modulations, then high absolute values in GM and DM should be paired with low absolute values of the SM, and vice versa. In PRR, absolute values of SM were negatively correlated with absolute values of both GM $(r=-0.3$; $p=0.005$; Spearman's rank correlation $)$ and DM $(r=-0.32 ; p=$ 0.003) (Fig. $7 d-f$ ). In PMd, only SM and DM were negatively correlated $(r=-0.36 ; p=0.006)$.

High absolute GM values do not necessarily mean that the neurons have to be gain modulated in an "ideal" way (i.e., in a multiplicative manner, as depicted in the example of Fig. $3 a$ ). Neurons with substantial GM might also have a high absolute SM value, which means, in principle, they might not have the typical unimodal directional tuning. In fact, a high absolute GM indicates a weak response of the neuron in either pro-reaches or anti-reaches. This can lead to weak or undefined directional tuning in the nonresponsive condition, and hence to high SM values. The lack of correlation between GM and SM, together with the anticorrelation of absolute GM and SM values in PRR, indicates that such interdependencies of GM and SM were not very common. To identify those cells that fulfilled all criteria for an ideal contextual gain modulation, we used an additional strict set of constraints that is compatible with the idea of gain modulation proper. We found that $48 \%$ (44 of 92) of cells that had a signifi- 
cant GM were (1) tuned in both context conditions, but did not have a significant SM and did not show a change in preferred direction, or (2) were only tuned in the corresponding context condition (i.e., in the pro condition if they had a positive $\mathrm{GM}$, or in the anti conditions if they had a negative GM) (PRR, 26 of 43, 60\%; PMd, 18 of $49,37 \%$ ).

\section{Discussion}

We found strong modulation effects of behavioral context on the predominant motor-goal tuning in PRR and PMd. We propose gain modulation as a mechanism to achieve flexible goal-directed visuomotor remapping in a context-specific manner. Second, we found different types of modulation with complementary biases in PRR and PMd. PRR showed stronger directional selectivity during the planning of reaches toward directly cued (pro) compared with inferred (anti) goals. PMd, however, showed stronger overall activity during reaches toward inferred compared with directly cued goals.

\section{Contextual modulations in PRR and PMd}

Behavioral context affected neural activity in PRR and PMd in two major ways, either by directly driving neurons (direct context modulation, DM) or by modulating spatial motor-goal representations (contextual gain/selectivity modulations, GM/ $\mathrm{SM}$ ). Both types of context modulation can be predicted from the hidden-layer properties of a neural network model (Brozović et al., 2007). Gain-field modulation evolved in this model as a consequence of learning context-specific spatial cue-response mapping, equivalent to our anti-reach task. Gain modulation of spatially selective neurons is the key principle underlying the spatial transformation mechanism in this model, similar to previous models of multisensory integration for spatial reference frame transformations (Zipser and Andersen, 1988; Salinas and Abbott, 1996). Gain modulation during multisensory integration for eye or hand movements had previously been found in the posterior parietal cortex (Andersen et al., 1985; Brotchie et al., 1995; Galletti et al., 1995; Snyder et al., 1998; Batista et al., 1999; Nakamura et al., 1999; Buneo et al., 2002) and frontal areas (Boussaoud et al., 1993, 1998; Mushiake et al., 1997; Cisek and Kalaska, 2002). In contrast, here we have shown gain modulation effects in PRR and PMd for remapping visuospatial information onto reach motor goals according to abstract cognitive transformation rules. a
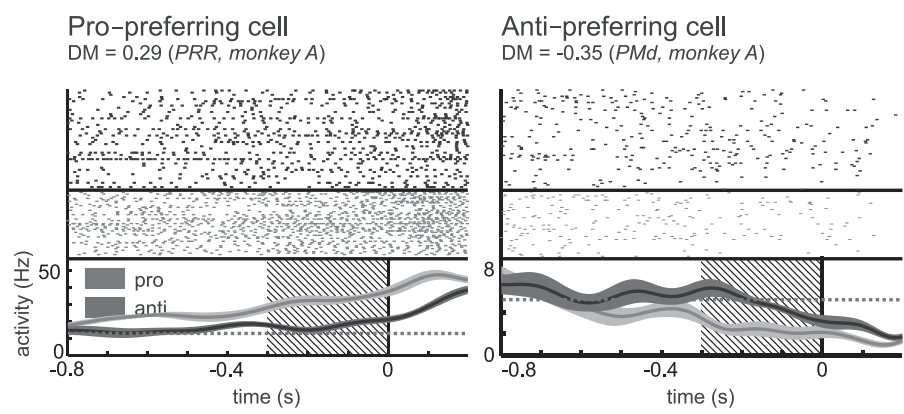

b Direct contextual modulation (DM)

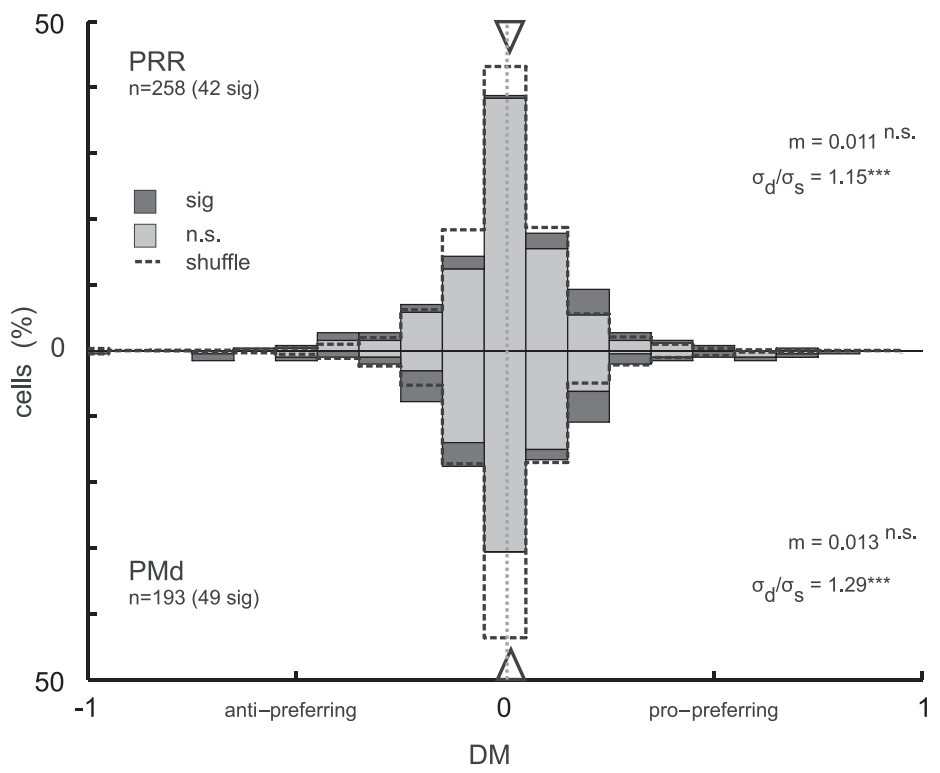

Figure 5. Direct context modulation in PRR and PMd independent of spatial tuning. $\boldsymbol{a}$, Examples show a pro-preferring cell (left), which was continuously more active in the memory period of pro-trials than during anti-trials ( $R$ condition). In the antipreferring cell (right), the contextual modulation appeared later (i.e., only $\sim 500 \mathrm{~ms}$ before the go cue). $\boldsymbol{b}$, Distribution of DM across the neuron populations. In PRR (top) as in PMd (bottom), the distributions were unbiased (i.e., centered at zero). The distribution of DM in both areas was broader than that of the shuffled data (dashed line; $\sigma_{\mathrm{d}}>\sigma_{\mathrm{s}^{\prime}}$ Bartlett's test) indicating contextual modulation effects (all conventions are as in Fig. 3).
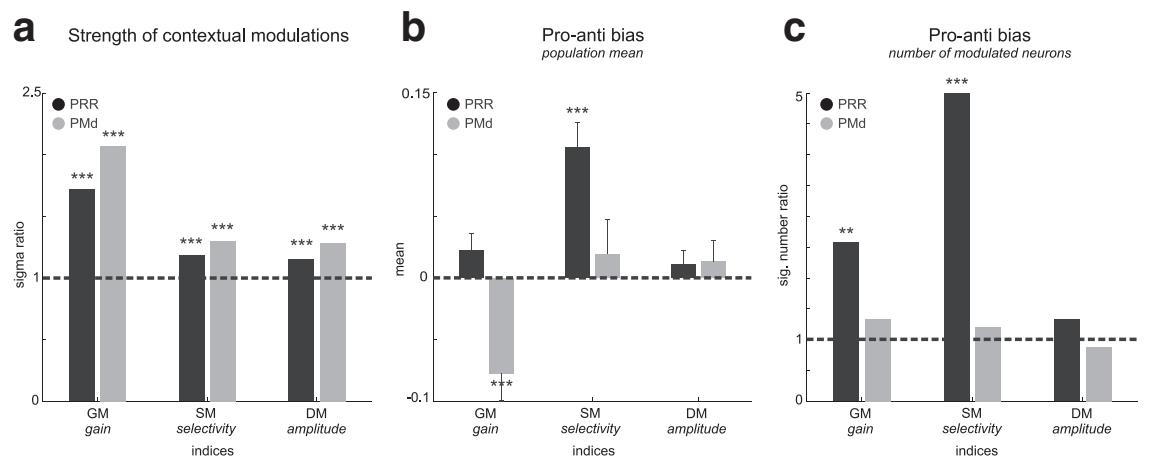

Figure 6. Summary of the strength and bias of contextual modulations in PRR (black) and PMd (gray). $\boldsymbol{a}$, Strength of contextual modulations. Both areas showed contextual modulations of all three types (GM, SM, and DM; Bartlett's test; significance levels are as in Fig. 3), with GM being the strongest. The $\sigma$ ratio indicates the width of the distribution of modulation indices relative to the width of the distribution of the shuffled data (see Materials and Methods) (Figs. 3-5). $\boldsymbol{b}$, Pro/anti bias in the population mean. PRR and PMd showed complementary pro/anti biases in the mean contextual gain and selectivity modulation (GM and SM; mean \pm SEM; $t$ test). $c$, Pro/anti bias in the number of modulated neurons. The relative number of neurons with a propreference versus an antipreference was higher in PRR for GM and SM, but balanced otherwise $\left(\chi^{2}\right.$ test). 
a

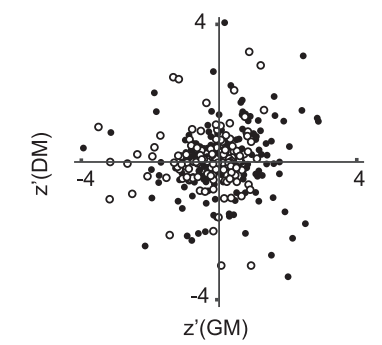

- PRR $r=0.1 \quad(p=0.17)$

d
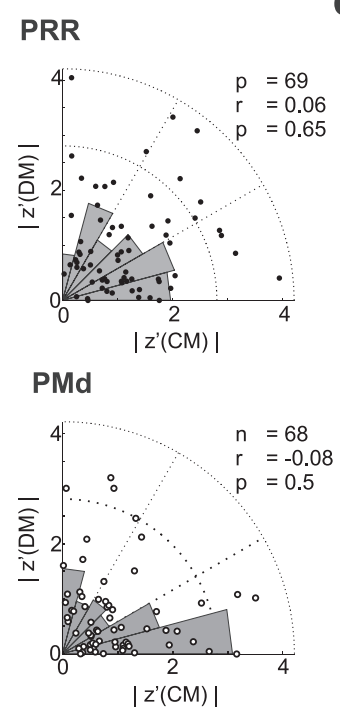

b

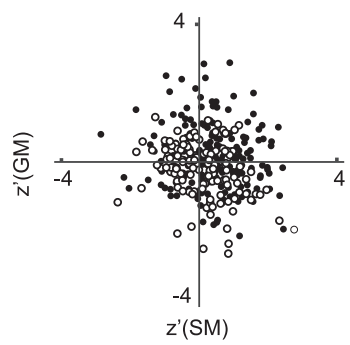

PRR $r=-0.13(p=0.07)$
PMd $r=-0.07(p=0.42)$

e
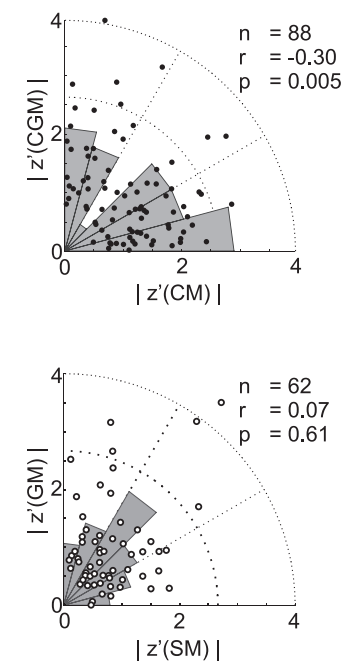

C

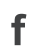

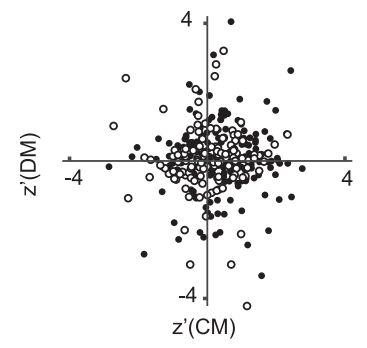

PRR $r=0.06(p=0.4)$
PMd $r=-0.03(p=0.7)$
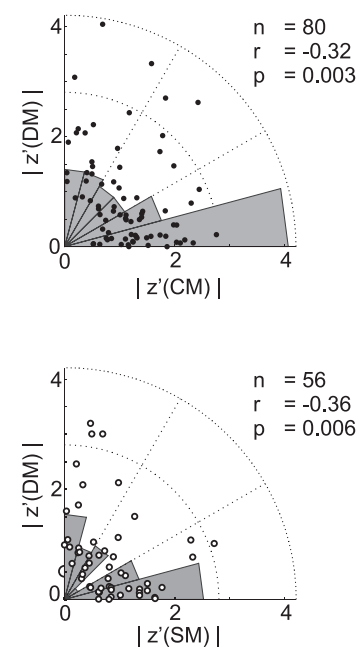

Figure 7. Interdependence of contextual modulations in PRR and PMd. $\boldsymbol{a}-\boldsymbol{c}$, Pairwise cross-correlations between the three modulation indices. The Pearson correlation of the three modulation indices ( $r$ and $p$ values given at the bottom of the diagrams) across neurons shows that none of the indices in either PRR or PMd were significantly correlated. $\boldsymbol{d}-\boldsymbol{f}$, Pairwise cross-correlation of the absolute values of the modulation indices for PRR (top) and PMd (bottom) separately. In PRR, SM-DM (f; top) and SM-GM ( $\boldsymbol{e}$; top) were negatively correlated [i.e., strong GM and DM modulations implied weak SM modulations, and vice versa (Spearman's rank correlation)]. In PMd, only SM and DM showed negative correlations $(\boldsymbol{f})$. The underlying rose plots (gray circular histograms) illustrate the frequencies of relative modulation strengths.

Gain modulation of neurons with motor-goal tuning was partly accompanied by modulation of the directional selectivity. This is consistent with the idea that a contextual gain modulation network builds the computational basis for the remapping process. Gain modulation then does not imply multiplicative modulations in the strict mathematical sense, but rather a summation of bell-shaped tuning functions (directional tuning) with a gain function (contextual tuning), which is passed through a recurrent network with nonlinear transfer properties (Salinas and Abbott, 1996; Brozović et al., 2007, 2008). Such a computational architecture results in gain modulations, which may not only affect the amplitude but also the shape of the tuning functions. This can be observed to varying extend for neurons within the same sensorimotor layer (Brozović et al., 2007).

Direct context modulation, as observed during the $\mathrm{R}$ condition, is also very plausible in the light of a gain modulation network. It is predicted for the sensorimotor ("hidden") layer of the network model, when only contextual information but no spatial information is available (Brozović et al., 2007), and all inputs are a priori additive. The impact of the context input in directly driving the neurons might be relatively weak, and only unfold to its full modulatory potential when the neuron is additionally

driven by strong spatial input (Abbott and Chance, 2005). Because of their latency (Fig. 5, right example), we do not attribute the direct context modulations to the sensory properties of the context cue, but rather to the behavioral relevance of the cue (Boussaoud and Wise, 1993; Snyder et al., 1997; Toth and Assad, 2002; Stoet and Snyder, 2004; Nakayama et al., 2008).

Neurons in PMd had previously been reported to be modulated by context, since task rules had an effect on spatial selectivity (di Pellegrino and Wise, 1993; Crammond and Kalaska, 1994). Spatially selective responses were shown to be dependent on whether the motor goal was at the same position as the visual cue or at a default location (di Pellegrino and Wise, 1993). In contrast to our data, the strong condition dependency in the study of di Pellegrino and Wise is attributable to the fact that, in the default condition, the visual cue did not contain any relevant spatial information. Hence the modulation did not reflect an effect of contextualspecific spatial remapping, but rather an effect of motor planning based on spatial remapping versus nonspatial stimulusresponse associations (Crammond and Kalaska, 2000). In contrast, in our experiment, the visual cue location was always relevant for the reach direction and the context modulations are the consequence of two different spatial transformation rules. Crammond and Kalaska reported a substantial fraction of cells in PMd with a significant interaction effect of a remapping rule on the reach-related response during the late phase of an instructed delay period (Crammond and Kalaska, 1994). Their finding could reflect a similar gain mechanism as observed here. However, they could not analyze specific effects of context on gain or selectivity of directional tuning because of their experimental protocol. Also, both previous studies could not compare the effects in PMd with PRR, in which gain modulation effects on directional tuning by spatial transformation rules, to our knowledge, have not been shown before.

\section{Differences between PRR and PMd}

Spatial motor-goal tuning in PRR and PMd, in part, was differently affected by contextual modulations. First, PMd neurons were on average stronger active by $15 \%$ during planning of antireaches, but only if spatial information was already available, i.e., in the RS but not in the R condition, negative bias of GM, but not $\mathrm{DM}$. We interpret this as an indication for the need of overruling a "default" movement plan (pro-reach) induced by the visual cue (Schlag-Rey et al., 1997; Everling et al., 1999), which might otherwise dominate. The stronger activity in PMd during antireaches in this view reflects the learned counterbalance of the network, which is necessary to compensate for the imbalance between pro-reaches and anti-reaches in terms of cue-response congruency. The counterbalance is only necessary in conditions 
in which a spatial cue was already presented, to suppress a default pro-movement (RS condition), but not when only the rule is known and no specific default plan yet exists (R condition). Enhanced activation because of spatial cue-response incongruence was found previously in the supplementary eye fields during the planning of antisaccades (Schlag-Rey et al., 1997; Amador et al., 2004). Stronger responses during planning of prosaccades compared with antisaccades, however, were found in the posterior parietal area LIP (lateral intraparietal area) (Gottlieb and Goldberg, 1999). We found a higher fraction of individual neurons in PRR with a pro-preferring bias in response strength, but no bias in the average population response.

Second, neurons in PRR were more directionally selective during planning of pro-reaches (positive bias of SM corresponding to 20\% stronger selectivity). This might reflect conflicting input to PRR specifically during planning of anti-reaches. Opposing input could result from bottom-up visuospatial information about the (remembered) cue location or default reach goal, on the one hand, and top-down projections of spatial motor-goal information from motor-tuned output stages, on the other hand (Brozović et al., 2007). Despite predominant motor-goal tuning on the population level (Gail and Andersen, 2006), the combination of such opposing inputs could explain the strongly reduced directional selectivity, which was found in many PRR neurons mainly in the anti-reach condition.

Together, the complementary effects of context on motorgoal representations indicate that posterior parietal cortex might more strongly represent stimulus-driven default or automatic movement plans (Desmurget et al., 1999; Pisella et al., 2000), whereas premotor cortex counterbalances this with predominant representations of rule-guided "inferred" movement plans.

\section{Different contextual modulations in different neuronal populations}

In PRR, those neurons that show strong gain modulation (high absolute GM) tended to have weak directional selectivity modulation (low absolute SM), and vice versa. This suggests that gain and selectivity modulations are the result of two separate underlying mechanisms, which affect different neuronal subpopulations. Gain and direct modulations, on the other hand, did not show such mutual exclusiveness. These findings are consistent with our predictions based on the previous model (Brozović et al., 2007). If the reduced directional selectivity in anti-reaches is indeed a consequence of conflicting independent bottom-up visuospatial and top-down motor-goal input to PRR, then we would not expect the GM/DM versus SM to necessarily be large in the same neurons. In PMd, we could not identify a systematic relationship or correlation pattern between the different types of contextual modulations.

\section{Gain modulation as universal mechanism for flexible remapping}

The contextual gain modulation of motor-goal representations in PMd and PRR strongly suggests gain-field mechanisms for flexibly mapping spatial sensory information onto spatial motorgoal representations according to arbitrary transformation rules. Based on our data, we propose that gain modulation is used by the brain not only in sensorimotor areas to achieve reference frame transformations driven by multisensory input (Andersen et al., 1985; Zipser and Andersen, 1988; Boussaoud et al., 1993, 1998; Brotchie et al., 1995; Galletti et al., 1995; Buneo et al., 2002), but also in the frontoparietal reach network to achieve contextually modulated, goal-directed visuomotor remapping, as previ- ously suggested theoretically (Salinas, 2004; Brozović et al., 2007). Our current data support this idea by providing first experimental evidence for context-specific gain modulations of spatial motor-goal tuning in PRR and PMd, which could denote the key underlying principle of flexible goal-directed behavior.

\section{References}

Abbott LF, Chance FS (2005) Drivers and modulators from push-pull and balanced synaptic input. Prog Brain Res 149:147-155.

Amador N, Schlag-Rey M, Schlag J (2004) Primate antisaccade. II. Supplementary eye field neuronal activity predicts correct performance. J Neurophysiol 91:1672-1689.

Andersen RA, Buneo CA (2002) Intentional maps in posterior parietal cortex. Annu Rev Neurosci 25:189-220.

Andersen RA, Essick GK, Siegel RM (1985) Encoding of spatial location by posterior parietal neurons. Science 230:456-458.

Batista AP, Buneo CA, Snyder LH, Andersen RA (1999) Reach plans in eyecentered coordinates. Science 285:257-260.

Blatt GJ, Andersen RA, Stoner GR (1990) Visual receptive field organization and cortico-cortical connections of the lateral intraparietal area (area LIP) in the macaque. J Comp Neurol 299:421-445.

Boussaoud D, Wise SP (1993) Primate frontal-cortex-effects of stimulus and movement. Exp Brain Res 95:28-40.

Boussaoud D, Barth TM, Wise SP (1993) Effects of gaze on apparent visual responses of frontal-cortex neurons. Exp Brain Res 93:423-434.

Boussaoud D, Jouffrais C, Bremmer F (1998) Eye position effects on the neuronal activity of dorsal premotor cortex in the macaque monkey. J Neurophysiol 80:1132-1150.

Brotchie PR, Andersen RA, Snyder LH, Goodman SJ (1995) Head position signals used by parietal neurons to encode locations of visual stimuli. Nature 375:232-235.

Brozović M, Gail A, Andersen RA (2007) Gain mechanisms for contextually guided visuomotor transformations. J Neurosci 27:10588-10596.

Brozović M, Abbott LF, Andersen RA (2008) Mechanism of gain modulation at single neuron and network levels. J Comput Neurosci 25:158-168.

Buneo CA, Jarvis MR, Batista AP, Andersen RA (2002) Direct visuomotor transformations for reaching. Nature 416:632-636.

Cisek P (2007) Cortical mechanisms of action selection: the affordance competition hypothesis. Philos Trans $\mathrm{R}$ Soc Lond B Biol Sci 362:1585-1599.

Cisek P, Kalaska JF (2002) Modest gaze-related discharge modulation in monkey dorsal premotor cortex during a reaching task performed with free fixation. J Neurophysiol 88:1064-1072.

Colby CL, Gattass R, Olson CR, Gross CG (1988) Topographical organization of cortical afferents to extrastriate visual area PO in the macaque-a dual tracer study. J Comp Neurol 269:392-413.

Crammond DJ, Kalaska JF (1994) Modulation of preparatory neuronal activity in dorsal premotor cortex due to stimulus-response compatibility. J Neurophysiol 71:1281-1284.

Crammond DJ, Kalaska JF (2000) Prior information in motor and premotor cortex: activity during the delay period and effect on pre-movement activity. J Neurophysiol 84:986-1005.

Desmurget M, Epstein CM, Turner RS, Prablanc C, Alexander GE, Grafton ST (1999) Role of the posterior parietal cortex in updating reaching movements to a visual target. Nat Neurosci 2:563-567.

di Pellegrino G, Wise SP (1993) Visuospatial versus visuomotor activity in the premotor and prefrontal cortex of a primate. J Neurosci 13:1227-1243.

Everling S, Dorris MC, Klein RM, Munoz DP (1999) Role of primate superior colliculus in preparation and execution of anti-saccades and prosaccades. J Neurosci 19:2740-2754.

Gail A, Andersen RA (2006) Neural dynamics in monkey parietal reach region reflect context-specific sensorimotor transformations. J Neurosci 26:9376-9384.

Galletti C, Battaglini PP, Fattori P (1995) Eye position influence on the parieto-occipital area PO (V6) of the macaque monkey. Eur J Neurosci 7:2486-2501.

Gottlieb J, Goldberg ME (1999) Activity of neurons in the lateral intraparietal area of the monkey during an antisaccade task. Nat Neurosci 2:906-912.

Kalaska JF (1996) Parietal cortex area 5 and visuomotor behavior. Can J Physiol Pharmacol 74:483-498. 
Marconi B, Genovesio A, Battaglia-Mayer A, Ferraina S, Squatrito S, Molinari M, Lacquaniti F, Caminiti R (2001) Eye-hand coordination during reaching. I. Anatomical relationships between parietal and frontal cortex. Cereb Cortex 11:513-527.

Mushiake H, Tanatsugu Y, Tanji J (1997) Neuronal activity in the ventral part of premotor cortex during target-reach movement is modulated by direction of gaze. J Neurophysiol 78:567-571.

Nakamura K, Chung HH, Graziano MS, Gross CG (1999) Dynamic representation of eye position in the parieto-occipital sulcus. J Neurophysiol 81:2374-2385.

Nakayama Y, Yamagata T, Tanji J, Hoshi E (2008) Transformation of a virtual action plan into a motor plan in the premotor cortex. J Neurosci 28:10287-10297.

Pisella L, Gréa H, Tilikete C, Vighetto A, Desmurget M, Rode G, Boisson D, Rossetti Y (2000) An "automatic pilot" for the hand in human posterior parietal cortex: toward reinterpreting optic ataxia. Nat Neurosci 3:729-736.

Rushworth MF, Nixon PD, Passingham RE (1997) Parietal cortex and movement. 1. Movement selection and reaching. Exp Brain Res $117: 292-310$

Salinas E (2004) Fast remapping of sensory stimuli onto motor actions on the basis of contextual modulation. J Neurosci 24:1113-1118.

Salinas E, Abbott LF (1996) A model of multiplicative neural responses in parietal cortex. Proc Natl Acad Sci U S A 93:11956-11961.

Salinas E, Thier P (2000) Gain modulation: a major computational principle of the central nervous system. Neuron 27:15-21.

Schlag-Rey M, Amador N, Sanchez H, Schlag J (1997) Antisaccade perfor- mance predicted by neuronal activity in the supplementary eye field. $\mathrm{Na}$ ture 390:398-401.

Snyder LH, Batista AP, Andersen RA (1997) Coding of intention in the posterior parietal cortex. Nature 386:167-170.

Snyder LH, Grieve KL, Brotchie P, Andersen RA (1998) Separate body- and world-referenced representations of visual space in parietal cortex. Nature 394:887-891.

Stoet G, Snyder LH (2004) Single neurons in posterior parietal cortex of monkeys encode cognitive set. Neuron 42:1003-1012.

Toni I, Rushworth MF, Passingham RE (2001) Neural correlates of visuomotor associations-spatial rules compared with arbitrary rules. Exp Brain Res 141:359-369.

Toth LJ, Assad JA (2002) Dynamic coding of behaviourally relevant stimuli in parietal cortex. Nature 415:165-168.

Wallis JD, Miller EK (2003) From rule to response: neuronal processes in the premotor and prefrontal cortex. J Neurophysiol 90:1790-1806.

Weinrich M, Wise SP (1982) The premotor cortex of the monkey. J Neurosci 2:1329-1345.

Wise SP, Murray EA (2000) Arbitrary associations between antecedents and actions. Trends Neurosci 23:271-276.

Wise SP, Boussaoud D, Johnson PB, Caminiti R (1997) Premotor and parietal cortex: corticocortical connectivity and combinatorial computations. Annu Rev Neurosci 20:25-42.

Zar JH (1999) Biostatistical analysis. Upper Saddle River, NJ: Prentice Hall.

Zipser D, Andersen RA (1988) A back-propagation programmed network that simulates response properties of a subset of posterior parietal neurons. Nature 331:679-684. 